\title{
LAPAROSCOPIC VERSUS OPEN ABDOMINOPERINEAL RESECTION FOR LOW RECTAL AND ANAL CARCINOMA- A SINGLE INSTITUTIONAL EXPERIENCE
}

\author{
Shailesh Patel ${ }^{1}$, Rohit Kumar Jha ${ }^{2}$, Rajan A Tankshali ${ }^{3}$, Rushit Dave ${ }^{4}$,Mahavir Tadaiya ${ }^{5}$, Kiran C Kothari ${ }^{6}$, Vikash Warikoo $^{7}$
}

${ }_{1}^{1}$ Assistant Professor, Department of Oncosurgery, Gujarat Cancer \& Research Institute, Ahmedabad.

${ }_{2}^{2}$ MCH Resident, Department of Oncosurgery, Gujarat Cancer \& Research Institute, Ahmedabad.

3 Professor, Department of Oncosurgery, Gujarat Cancer \& research Institute, Ahmedabad.

${ }^{4}$ MCH Resident, Department of Oncosurgery, Gujarat Cancer \& Research Institute, Ahmedabad.

${ }_{5}^{5}$ Assistant Professor, Department of Oncosurgery, Gujarat Cancer \& Research Institute, Ahmedabad.

${ }^{6}$ Deputy Director, Department of Oncosurgery, Gujarat Cancer \& Research Institute, Ahmedabad.

${ }^{7}$ Associate Professor, Department of Oncosurgery, Gujarat Cancer \& Research Institute, Ahmedabad.

\section{ABSTRACT}

\section{BACKGROUND}

There are various studies delineating that laparoscopic colonic surgery results in less postoperative pain, rapid recovery, shorter hospital stay, and quick resumption of daily activities when compared to open surgery. But there are only few studies comparing the laparoscopic assisted to open abdominoperineal resection for low rectal or anal canal cancers.

The aim of this study is to evaluate and compare the results of laparoscopic assisted and open abdominoperineal resection (LapAPR and Open-APR) for low rectal cancer and anal canal cancer.

\section{MATERIALS AND METHODS}

Between $1^{\text {st }}$ October 2012 to 30th September 2015 at Gujarat Cancer \& Research Institute, 82 patients underwent surgery for low rectal adenocarcinoma or anal carcinoma. Among them, 37 patients underwent laparoscopic APR and 45 patients underwent open APR. The operative outcomes and postoperative complications of these two groups were compared.

\section{RESULTS}

There were no significant differences between Lap-APR and open-APR in patient's age and tumour stage. Post-operative recovery was better in Lap-APR group, with earlier return of bowel function and early mobilisation more significant. But the operative time was longer in Lap-APR. Perineal wound morbidity was same in both groups, but the abdominal wound infection rate was higher in Open-APR, as Lap-APR with no long laparotomy wounds will only have small Trocar wounds. Two-year survival was nearly same in both groups.

\section{CONCLUSION}

Lap-APR has advantages over Open-APR in rapid postoperative recovery of bowel function, early oral intake, and early mobilisation, but Lap-APR has longer operative time. The operating time of laparoscopic APR has also decreased over time.

\section{KEYWORDS}

Laparoscopic Abdominoperineal Resection, Open Abdominoperineal Resection.

HOW TO CITE THIS ARTICLE: Patel S, Jha RK, Tankshali RA, et al. Laparoscopic versus open abdominoperineal resection for low rectal and anal carcinoma- A single institutional experience. J. Evolution Med. Dent. Sci. 2017;6(61):4512-4514, DOI: $10.14260 /$ Jemds/2017/975

\section{BACKGROUND}

Abdominoperineal Resection (APR) is the surgical procedure done on patients with distal rectal cancer in which an anterior resection cannot be done to preserve anal sphincter or for anorectal cancer..$^{1,2}$

APR was first described by Ernest Miles in 1908, and Jacobs was the first who, in 1991 reported the Laparoscopic colectomy. Since then Laparoscopic colorectal surgery is being increasingly practised worldwide. ${ }^{1}$

Recently APR was performed in not more than $14 \%$ of patients of rectal cancer. ${ }^{3}$ With laparoscopic technique for

Financial or Other, Competing Interest: None.

Submission 12-06-2017, Peer Review 06-07-2017,

Acceptance 12-07-2017, Published 31-07-2017.

Corresponding Author:

Dr. Rohit Kumar Jha,

Flat - 404, Atulyam-3,

IGP Campus, Near NIOH,

Meghaninagar, Ahmedabad-380016.

E-mail: wihrohitjha@gmail.com

DOI: $10.14260 /$ jemds $/ 2017 / 975$

\section{(c) (i) $(3)$}

colon and rectum resection, patients have less postoperative pain, shortened postoperative ileus, reduced duration of hospital stay with rapid recovery and quick resumption of normal daily activities. ${ }^{4,5}$ In laparoscopic APR, the magnified view of narrow pelvis facilitates identification of surgical planes and nerves. ${ }^{6}$ The purpose of this study was to evaluate and compare the results of laparoscopic and open abdominoperineal resection for low rectal adenocarcinoma and anal carcinoma, the postoperative recovery, complications.

\section{MATERIALS AND METHODS}

This prospective study was done on 82 patients, having low rectal cancer (within $5 \mathrm{~cm}$ of anal verge) and anal carcinoma admitted and operated (Laparoscopic APR/Open APR) from $1^{\text {st }}$ October 2012 to $30^{\text {th }}$ September 2015. Of these 82 patients, 37 patients underwent laparoscopic assisted abdominoperineal resection and placed in Lap-APR group, and 45 patients underwent open abdominoperineal resection, and placed in Open-APR group. Five patients were started as LAP but due to severe adhesion they were 
converted into Open, they were also grouped in 'Open' category. Decision about the technique (Lap-APR or Open APR) is made by the operating surgeon with consultation with patients. All patients gave written informed consent preoperatively.

\section{Patients having following criteria were excluded from the study \\ a. Tumour more than $5 \mathrm{~cm}$ higher up from anal verge, \\ b. Bulky tumour or locally advanced tumour, \\ c. Patients having multiple cancers or synchronous proximal colonic cancer, \\ d. Patients having ulcerative colitis, \\ e. Patients with distant metastasis, \\ f. Patients having significant comorbidities (MI, DM, renal failure, chronic liver disease), \\ g. Patients presenting with recurrent rectal cancer.}

All patients after physical examination underwent preoperative proctoscopy and complete colonoscopy and biopsy of the tumour, abdominal and pelvis ultrasonography and computed tomography to record the size of tumour and involvement of adjacent structures, and to see the secondaries in liver, chest X-Ray to see the lung metastasis. Complete blood count and carcinoembryonic antigen tests were conducted before surgery. Patients with tumour stage $\mathrm{T} 3$ and regional lymph node enlargement were offered preoperative chemoradiotherapy (CCRT). Mechanical bowel preparation was carried out day before surgery with sodium phosphate oral solution. Cefo-sulbactam 1.5 G was administered intravenously at induction of anaesthesia. Urinary bladder was routinely catheterised. All operations were done under general anaesthesia and patients were placed in modified lithotomy position. In a Lap-APR technique, surgeon stands right to the table, monitor and assistant stands left side. For perineal part of operation, surgeon stands/sits in between the leg rest of the table. Pneumoperitoneum is created by open technique and $10 \mathrm{~mm}$ trocar is inserted below the umbilicus. Three or four working trocars are inserted under direct vision in the right and left midclavicular line at the level of umbilicus and anterior superior iliac spine. The left lower Trocar is inserted in left lower quadrant at the planned site of colostomy. The sigmoid colon and rectum are mobilised by using medial and lateral approach. Inferior mesenteric artery $1.5 \mathrm{~cm}$ above its origin is clipped and divided. The ureter, the hypogastric nerve and the pelvic parasympathetic plexus are preserved. Rectum and whole mesorectum is completely mobilised, the sigmoid colon is transected with linear stapler and the specimen is removed through the perineal wound. An end colostomy is constructed at the left lower trocar site. The perineal wound is closed after placing a drain in the pelvic cavity through a separate stab wound. The Open-APR was performed by midline laparotomy incision, otherwise same as Lap-APR. Operative outcomes were recorded and compared between the two groups.

\section{RESULTS}

Eighty-two patients were operated for adenocarcinoma of low rectum. 37 (45.12\%) patients were operated by Lap-APR technique, among them $23(62 \%)$ patients were male, and 14 $(38 \%)$ patients were female. $45(54.88 \%)$ patients were operated by Open-APR technique, among them 29 (64.45\%) patients were male, while $16(35.5 \%)$ patients were female. There were 5 patients who were converted from Lap-APR to Open-APR technique. Most common reason for conversion was dense adhesion in pelvis.

About operative outcomes, the mean operative time was slightly longer in Lap-APR (175 minutes), while in Open-APR it is 155 minutes. The time to pass first bowel movement was significantly less in Lap-APR (mean 56.4 hours) while in Open-APR it was 68 hours (mean).

Patients in Lap-APR group started taking water earlier than patients of Open-APR group $(41 \pm 13.2$ hours in Lap-APR $54 \pm 12.2$ hours in Open-APR), which is statistically significant.

After surgery, patients of Lap-APR group started taking soft diet earlier than Open-APR group (4.6 \pm 1.2 days in LapAPR and $5.5 \pm 1.7$ days in Open-APR), that is also more significant.

Patients of Lap-APR were mobilised early, $6.9 \pm 3.19$ days in Lap-APR while $9.2 \pm 3.45$ days in Open-APR. Postoperative hospital stay was slightly less in Lap-APR than in Open-APR group, $14.8 \pm 3.4$ days in Lap APR and $16.5 \pm 4.8$ days in Open-APR. 3 (8\%) patients in LAP group developed subacute intestinal obstruction, all of them managed conservatively, while $8(17 \%)$ patients in Open-APR group developed intestinal obstruction in post-operative period, out of which in 3 patients $(6 \%)$ exploratory laparotomy was done and rest were managed conservatively.

In Open-APR group, 6 patients developed abdominal wound infection, which is significant. 6 males $(16.2 \%)$ and 3 females (8\%) in Lap-APR while 7 males (15.5\%) and 5 females $(11 \%)$ in Open-APR described that their sexual function worsened.

The rate of tumour recurrence was similar in both groups.

Two-year survival was $91.9 \%$ in Lap-APR and $91.1 \%$ in Open-APR group. Local recurrence occurred in $2(6.7 \%)$ and $3(6.7 \%)$ patients of Lap-APR and open-APR group respectively.

Liver recurrences occurred in $4(10.8 \%)$ patients of LapAPR and $5(11.1 \%)$ patients of Open-APR.

\section{DISCUSSION}

Laparoscopic assisted abdominoperineal resection was first described by the Sackier in 1992. After that many studies have demonstrated the benefits and safety of laparoscopic rectal surgery for rectal cancer. Decanini et al described in their study that Lap-APR can be performed according to oncologic principles with proximal vascular ligation of inferior mesenteric artery. This study demonstrates that the Lap-APR did not jeopardise patients' oncologic outcome.

Toe-WeiKe et al study described the same oncologic outcome, but some studies reported the risk of port site metastasis in Lap-APR. In this study, no port site metastasis occurred in any patient. ${ }^{7}$

Some studies showed that Lap-APR had better immediate outcomes in terms of fast return of bowel function, early mobilisation and less analgesic requirement, when compared with open surgery for rectal cancer. ${ }^{8}$ This study also shows better results of Lap-APR in terms of faster return of bowel function and shorter postoperative hospital stay duration, when compared to Open-APR. But the mean operative time was longer in Lap-APR, while it is short in Open-APR. Patients 
of Lap-APR were more comfortable, and were mobilised early as compared to Open-APR. Other studies also showed better mobilisation results of Lap-APR except longer operative time. .,10 $^{10}$

A study by Inomata $M$ et al reveals no significant shortening in the length of hospital stay in Lap-APR. ${ }^{11}$ Male and female sexual dysfunction after Lap- and Open-APR had no significant differences.

Quah H study shows poorer sexual outcomes in Lap-APR when compared to Open-APR. ${ }^{12}$

A study by Paraskevas et al elicited that sexual function was significantly worse one year after laparoscopic surgery. ${ }^{13}$

Patients in the Lap-APR group with no long abdominal laparotomy incisions except of trocar site, seem to provide early mobilisation and recovery, it also made easier to educate patients for stoma management. It also seems stoma care is easier without long abdominal incision in Lap-APR group. Open-APR cases had two big wounds, one long abdominal laparotomy wound, and a second perineal wound. In this way, APR is different from other colorectal resection, in having a higher complication rate because of the perineal wound. Although the Lap-APR is with no long laparotomy wound, the perineal wound and its related complications might not change by the Lap-APR. In addition to reduced abdominal wall trauma in Lap-APR, the less manipulation of abdominal contents may diminish postoperative adhesions and reduce the rate of incisional hernia. While in Open-APR there is more chance of postoperative adhesions, intestinal obstruction, and incisional hernia.

\section{CONCLUSION}

Lap-APR has particular advantages to patients with low rectal cancer, including rapid recovery of bowel function, early oral intake of water, semi-fluid and solid diet, and early education of stoma care. Stoma care is also easy in Lap-APR. Short hospital stay without jeopardising oncologic results is noted in Lap-APR, but at the expense of long operative time and more technically demanding procedure.

\section{REFERENCES}

[1] Perry WB, Connaughton JC. Abdominoperineal resection: how is it done and what are the results. Clinics Colon Rectal Surg 2007;20(3):213-20.
[2] Braga M, Vignali A, Gianotti L, et al. Laparoscopic versus open colorectal surgery: a randomized trial on short-term outcome. Ann Surg 2002;236(6):759-66.

[3] Baker RP, White EE, Titu L, et al. Does laparoscopic abdominoperineal resection of the rectum compromise long term survival? Dis Colon Rectum 2002;45(11):1481-5.

[4] Miles WE. A method of performing abdomino-perineal excision for carcinoma of the rectum and the terminal portion of the pelvic colon (1908). CA Cancer J Clin 1971;21(6):361-4.

[5] Wexner SD, Reissman P, Pfeifer J, et al. Laparoscopic colorectal surgery: analysis of 140 cases. Surg Endosc 1996;10(2):133-6.

[6] Tomita H, Marcello PW, Milsom JW. Laparoscopic surgery of the colon and rectum. World Journal of Surgery 1999;23(4):397-405.

[7] Tao-Wei K, Tzu-Liang WC, Ting-Ming $\mathrm{H}$, et al. Laparoscopic abdominoperineal resection for low rectal adenocarcinoma. J Soc Colon Rectal Surgeon 2008;19:63-70.

[8] Fodera M, Pello MJ, Atabek U, et al. Trocar site tumour recurrence after laparoscopic-assisted colectomy. J Laparoendosc Surg 1995;5(4):259-62.

[9] Wexner SD, Cohen SM. Port site metastases after laparoscopic colorectal surgery for cure of malignancy. Br J Surg 1995;82(3):295-8.

[10] Leung KL, Kwok SP, Lau WY, et al. Laparoscopicassisted abdominoperineal resection for low rectal adenocarcinoma. Surg Endosc 2000;14(1):67-70.

[11] Inomata M, Nakajima K, Kono $Y$, et al. Surgical outcomes of laparoscopic versus open abdominoperineal resection for anorectal cancer: a comparative study. Surgery Curr Ress 2014;4(3).

[12] Quah HM, Jayne DG, Eu KW, et al. Bladder and sexual dysfunction following laparoscopically assisted and conventional open mesorectal resection for cancer. $\mathrm{Br}$ J Surg 2009;89(12):1551-6.

[13] Paraskevas S, George E, Theodoropoulo J, et al. Prospective evaluation of sexual function after open and laparoscopic surgery for rectal cancer. Surg Endosc 2009;23(12):2665-74. 